Han et al., Afr J Tradit Complement Altern Med. (2013) 10(6):422-425

\title{
http://dx.doi.org/10.4314/ajtcam.v10i6.2
}

\section{A STUDY ON THE EFFECT OF AQUEOUS EXTRACT OF LOBELIA CHINENSIS ON COLON PRECANCEROUS LESIONS IN RATS}

\author{
Shao-Rong Han, Xiao-Yan Lv, Yan-Ming Wang*, Hai Gong, Cong Zhang, An-Na Tong, Ning Yan \\ Department of Radiation Oncology, Jinan Military General Hospital, Jinan 250031, China \\ ${ }^{\star}$ E-Mail: ymwang64@126.com
}

\begin{abstract}
This paper studies the effects of Lobelia chinensis on colon precancerous lesions and on colonic epithelial proliferation and apoptosis in DMH-induced rats. After two weeks of feeding, 50 Wistar rats were randomly divided into five groups, namely the normal group, model group, Lobelia chinensis low-dose group, medium-dose group and high-dose group. Lobelia chinensis was made into ACF model, and administered to experimental groups for 10 consecutive weeks. Control group was given equivalent amount of normal saline. After feeding for 10 weeks, the rats in each group were sacrificed and the changes in colonic ACF number of rats in experimental groups were observed, and the inhibition rates were calculated. The results showed that among the rats fed for $24 \mathrm{~h}$ and $48 \mathrm{~h}$, the number of apoptotic cells in colonic crypts of rats in DMH group did not differ significantly from the control group, while the difference was obvious between the control group and Lobelia chinensis treatment groups. The medium and high doses, that is, $0.45 \mathrm{~g} / \mathrm{kg}$ and $1.35 \mathrm{~g} / \mathrm{kg}$ can significantly inhibit ACF formation (P<0.01). The inhibition rates of low, medium and high doses were $8.12 \%, 59.42 \%$ and $65.44 \%$, respectively.
\end{abstract}

Keywords: Lobelia chinensis; DMH group; colonic epithelial

\section{Introduction}

Colorectal cancer is a malignancy with evident genetic predisposition. $25 \%$ of patients with colorectal cancer have a family history; $3 \%-5 \%$ of the cancer patients present obvious "hereditary colon cancer syndromes", including the familial adenomatous polyposis (FAP) and hereditary non-polyposis colorectal cancer (HNPCC) (Zhao, 2010). There are mainly three types of gene mutations that lead to colon cancer; they are oncogene mutation, tumour suppressor gene mutation and mismatch repair gene (MMR) mutation (Li et al., 2004). Preliminary studies have found that Lobelia chinensis has some effect on colon cancer. It is reported in the literature that the compound Shenqitang can inhibit the metastasis and progression of human colon cancer cells in nude mice, and Lobelia chinensis is one of its ingredients (compound Shenqitang: Radix Angelicae Sinensis, Radix Aucklandiae, Pericarpium Citri Reticulate, Radix Glycyrrhizae, Poria cocos, ginseng, Radix Astragali, Radix Notoginseng, Lobelia chinensis, Sculellaria barbata, Radix Aconiti Preparata, Radix Aconiti Kusnezoffii Preparata, Kusnezoffii fried Guya, Fructus Oryzae Germinatus, Fructus Hordei Germinatus) ( Shen et al., 2003). Therefore, this experiment selected the DMF-induced Wistar rat colon carcinogenesis model to observe the effect of aqueous extract of Lobelia chinensis on colon precancerous lesions in rats after administration.

\section{Materials}

Animals

The experimental animals included 200 SPF male Wistar rats, weighing 130 150g, provided by the Beijing Vital River Laboratory Animal Technology Co., Ltd., animal license No.: SCXK (Beijing) 2011-0356. The materials used are DMH, methylene blue, purchased from Sigma; Basic Red (Beijing Aoboxing Biotechnology Co., Ltd.); Satorius electronic balance, pH meter; Olympus inverted microscope, and dissecting microscope.

\section{Drugs and reagents}

Lobelia chinensis, purchased from Beijing Tongrentang Pharmacy, was identified by Professor Liu Ming of Jinan Military General Hospital. The specimen was placed at the centre lab of general hospital. DMF was purchased from Beijing Lvyuanbode Biotechnology Co., Ltd. 


\section{http://dx.doi.org/10.4314/ajtcam.v10i6.2}

\section{Methods}

\section{Preparation of drug}

$20 \mathrm{~g}$ of Lobelia chinensis was weighed, pulverised with a pulveriser, and passed through a 20-mesh sieve. Then, $15 \mathrm{~g}$ was weighed, placed in a $250 \mathrm{ml}$ round-bottomed flask, added with $150 \mathrm{ml}$ of distilled water, and heated to reflux for $2 \mathrm{~h}$. Drug of the same batch was extracted three times. The resulting three filtrates were combined, heated in a water bath, then completely dried in a vacuum oven at $80{ }^{\circ} \mathrm{C}$, and ground into a dry powder. $5 \mathrm{~g}$ of the dry powder was weighed and prepared into an aqueous solution of $15 \mathrm{mg} / \mathrm{ml}$, which was then used in the administration process.

\section{Grouping and administration methods}

200 rats were randomly divided into five groups, namely the normal group, model group, Lobelia chinensis low-dose group, medium-dose group and high-dose group. The low, medium and high doses of Lobelia chinensis were $0.15 \mathrm{~g} / \mathrm{kg}$., $0.45 \mathrm{~g} / \mathrm{kg}$ and $1.35 \mathrm{~g} / \mathrm{kg}$ respectively. The male Wistar rats were fed for two weeks after purchase. In the subsequent two weeks, the ACF model was prepared. 25 mg/kg DMH was subcutaneously injected to the neck and back of rats once a week, a total of two times, to produce aberrant crypt foci. The administration was begun in the fourth week from feeding, which was continued for five consecutive weeks. The concentration of EDTA-NaCl solution with a DMH is $3 \mathrm{~g} / \mathrm{L}, \mathrm{pH} 6.5$.

\section{Colonic crypt cell apoptosis experiment (Liu, 2011)}

3 weeks after administration, $25 \mathrm{mg} / \mathrm{kg} \mathrm{DMH}$ was injected subcutaneously to the neck and back of the rats once a week for a total of two times. $24 \mathrm{~h}$ and $48 \mathrm{~h}$ after the second injection, the rats were sacrificed, and the effect of Lobelia chinensis aqueous extract dry powder on colonic crypt cell apoptosis was observed.

\section{Frequency and spatial distribution of apoptotic and mitotic cells}

$4 \mathrm{~h}$ and $72 \mathrm{~h}$ after DMH injection, rats were anesthetised by intraperitoneal injection of $20 \%$ urethane. Their colons were removed by dissection, washed with Krebs Ringger buffer, and colon full-length was measured with ascending colon as the starting point and the end of descending colon as the ending point. $1 \mathrm{~cm}$-long colon segments were removed from the $55 \%$ and $90 \%$ positions of the colons as the midsection colon and descending colon specimens. All specimens were $0.5 \mathrm{~cm}$ thick, and were Feulgen stained. Muscular layers were removed and colonic crypts were isolated with micro-fine needle under the optical microscope $(\times 400)$. The number of apoptotic and mitotic nuclei in each colonic crypt were calculated. 10 20 colonic crypts were randomly selected from each rat for measurement. The longitudinal length of each colonic crypt was divided into 10 equal parts from the bottom to the opening, and the number of apoptotic and mitotic nuclei in each part was calculated

\section{Determination of aberrant crypt foci (ACF)}

The rats were anesthetised, laparotomized and their entire colons were removed. The colons were washed with Krebs Ringger buffer, followed by measurement of colon full-length. The procedure was similar to the "frequency and spatial distribution of apoptotic and mitotic cells" (Zhou et al., 2010). The entire colons were cut along the longitudinal axis with mucosal side up, fixed in $10 \%$ neutral formalin, and stained with $0.2 \%$ methylene blue. Under the light microscopy $(\times 40)$, ACF number was observed. And the number of colonic crypts contained in ACF of each colon was recorded. Inhibition rate $\%=$ (ACF of control group - ACF of experimental group) $/$ control group $\times 100 \%$. 
Han et al., Afr J Tradit Complement Altern Med. (2013) 10(6):422-425

\section{http://dx.doi.org/10.4314/ajtcam.v10i6.2}

\section{Results}

Effect on colonic crypt cell apoptosis (Dong et al., 2005; Zhou et al., 2010)

Table 1: Effect of aqueous extract of Lobelia chinensis on apoptosis of single colonic crypt cell in rat colon $(\mathrm{X} \pm \mathrm{s}, \mathrm{n}=6)$

\begin{tabular}{|c|c|c|c|c|c|}
\hline \multirow{2}{*}{\multicolumn{2}{|c|}{ Group }} & \multicolumn{2}{|c|}{ Midsection colon/each } & \multicolumn{2}{|c|}{ Descending colon/each } \\
\hline & & $24 \mathrm{~h}$ & $48 \mathrm{~h}$ & $24 \mathrm{~h}$ & $48 \mathrm{~h}$ \\
\hline Normal & & $1.36 \pm 0.12$ & $1.35 \pm 0.21$ & $0.92 \pm 0.13$ & $1.12 \pm 0.20$ \\
\hline DMH & & $1.45 \pm 0.15$ & $1.52 \pm 0.21$ & $1.49 \pm 0.18$ & $1.46 \pm 0.23$ \\
\hline \multirow{3}{*}{$\begin{array}{l}\text { Lobelia } \\
\text { chinensis }\end{array}$} & Low-dose & $1.71 \pm 0.23$ & $1.68 \pm 0.15$ & $1.69 \pm 0.12$ & $1.72 \pm 0.14$ \\
\hline & Medium-dose & $2.21 \pm 0.14$ & $2.11 \pm 0.23$ & $1.93 \pm 0.16$ & $1.72 \pm 0.13$ \\
\hline & High-dose & $2.75 \pm 0.16$ & $2.37 \pm 0.17$ & $2.52 \pm 0.13$ & $2.14 \pm 0.12$ \\
\hline
\end{tabular}

The experimental results found that in the rats reared for $24 \mathrm{~h}$ and $48 \mathrm{~h}$ the number of apoptotic cells in colonic crypts of rats in DMH group did not differ significantly from the control group. However, the difference was obvious between the control group and Lobelia chinensis treatment group of which the number of apoptotic cells corresponding to the medium and high doses was statistically significantly different compared with the control group, while the low dose only had a slight change, which was not significantly different. The analysis of spatial distribution of crypt apoptosis of the middle and distal colons found that the crypt apoptosis was significantly increased, and its distribution was relatively dense. The results are shown in Table 2.

Table 2: Effect of aqueous extract of Lobelia chinensis on colon precancerous lesions in rats

\begin{tabular}{ccccc}
\hline Group & $\mathrm{N}$ & Dose $/ \mathrm{g} \cdot \mathrm{kg}^{-1}$ & ACF number & Inhibition rate $(\%)$ \\
\hline Control & 10 & - & - & \\
DMH & 10 & & $38.2 \pm 2.2$ & \\
Lobelia & & & & \\
chinensis & & & & \\
Low-dose & 10 & 0.15 & $35.1 \pm 1.9$ & 8.12 \\
Medium-dose & 10 & 0.45 & $15.5 \pm 1.4$ & 59.42 \\
High-dose & 10 & 1.35 & $13.2 \pm 1.1$ & 65.44 \\
\hline
\end{tabular}

Among the aqueous solutions of three different concentrations prepared by the Lobelia chinensis aqueous extract powder, the inhibition rate of low-dose group was significantly lower than medium- and high-dose groups. The medium and high doses, i.e. $0.45 \mathrm{~g} / \mathrm{kg}$ and $1.35 \mathrm{~g} / \mathrm{kg}$ respectively, can significantly inhibit the formation of ACF $(\mathrm{P}<0.01)$. The inhibition rates of low, medium and high doses were $8.12 \%, 59.42 \%$ and $65.44 \%$, respectively, as shown in Figure 1.

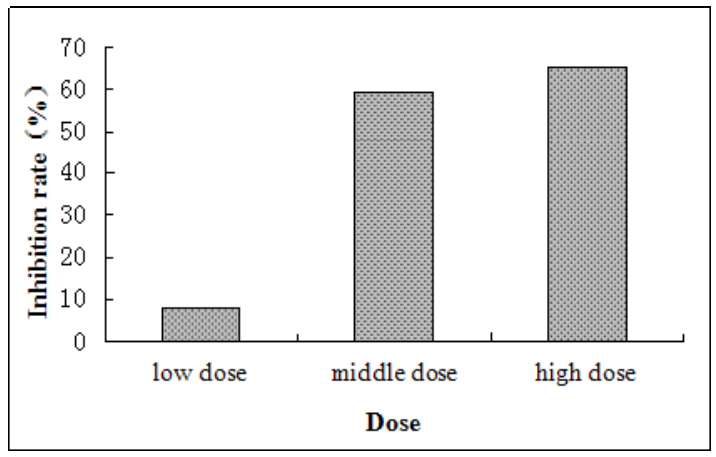

Figure 1: Inhibition rate of Lobelia chinensis aqueous extract on ACF

\section{Discussion}

At present, a number of Chinese medicinal extracts such as Venenum Bufonis, Radix Angelicae Sinensis, astragaloside and emodin all have good effects on colon cancer. For instance, Venenum Bufonis induces caspase-independent cell death in colon cancer cells as indicated by the 


\section{http://dx.doi.org/10.4314/ajtcam.v10i6.2}

absence of significant early apoptosis as well as PARP and caspase-3 cleavage. However, bufalin activated an autophagy that was linked to the generation of reactive oxygen species (ROS) and c-Jun NH2-terminal kinase (JNK) (Noriyuki et al., 2012), phthalides like n-butylidene phthalide, senkyunolide A and z-ligustilide extracted from Angelica sinensis exhibited selective cytotoxic and anti-proliferative effects in human colon cancer HT-29 cells. Total phthalide in the herbal extracts showed stronger antagonistic effects in CCD-18Co than in HT-29 cells (Winnie et al., 2008). In this paper, Wistar rats were used. The rats were randomly divided into five groups, namely the normal group, model group, Lobelia chinensis low-dose group, medium-dose group and high-dose group. The rats were intraperitoneally injected with DMH (1, 1-Dimethyl hydrazine) once a week, and administered for 10 consecutive weeks. Feeding was continued for 12 weeks, and then the rats were sacrificed. The changes in colonic ACF number of rats in experimental groups were observed and inhibition rates were calculated. By the determination of the effect of Lobelia chinensis aqueous extract on apoptosis of single colonic crypt cell in rat colon, it was found that the number of apoptotic cells in the medium and high-dose groups were statistically significantly different compared with the control group, while only a slight change was observed in the low dose group, where the difference was not obvious, indicating that Lobelia chinensis has some effects on both the prevention and treatment of colon cancer. Furthermore, the inhibition rates of Lobelia chinensis low, medium and high doses on ACF were $8.12 \%, 59.42 \%$ and $65.44 \%$, respectively.

The whole plant of Lobelia chinensis has the diuresis-inducing and oedema-alleviating, heat-clearing and toxic-eliminating effects, and it is commonly used in the treatment of acute nephritis, snake bites, herpes zoster, etc. For example, the Lobelia chinensis ethanol extract and its total alkaloids have a significant protective effect on rats infected with H9N2 and H1N1 subtype influenza viruses, which may be related to the respiratory stimulant effect of its alkaloids (Chen, 2010). As for the antitumour effects, the crude alkaloid extract of Lobelia chinensis has certain inhibitory effect on gastric cancer BG-38 cells, and the effect is strengthened with the increase of alkaloid concentration (Su et al., 2007). The cell staining and MTT colorimetric detections found that Lobelia chinensis also has a significant inhibitory effect on U266 cells, which is in a dose-dependent manner. DNA gel electrophoresis results revealed obvious DNA ladder (He \& Wu., 2012). The water decoction of Lobelia chinensis also has an inhibitory effect on the growth of hepatoma cells in mice, and the expression of P53 in $\mathrm{H} 22$ cells can be up-regulated by the action of the water decoction (Shao, 2010).

\section{References}

1. Chen Jian-xin. (2010) Studies on the Bioactive Constituents of Lobelia Chinensis. Supervisor: Ye Wen-cai. Jinan University.

2. Dong Li, Zhan Guang-xu, Huang Bo and Wu Da-zheng. (2005) Inhibitory effect of Jinfukang on precancerous lesions of colon adenocarcinoma in DMH-induced rats. Shandong Pharmaceutical Association. Proceedings of the First Annual Academic Conference of Shandong Pharmaceutical Association (1). Shandong Pharmaceutical Association: 5-6.

3. He Shan and Wu Guo-xin. (2012) Extract of alkaloids from Lobelia chinensis Lour and their inhibitory effects on growth of Myeloma Cells U266. Strait Pharmaceutical Journal, 09: 237-239.

4. Li Jun-sheng, Tang Wen-hao and Ji Zhen-ling. (2004) Genetic mechanisms of colorectal cancer. Journal of Clinical Medicine in Practice, 06: 10-12.

5. Liu Cong-cong. (2011) Expression of YAP and Its Relationship with the Apoptosis in the Colorectal Adenocarcinoma Tissues. Shanxi Medical University.

6. Noriyuki T., Naoko K., Terukazu I., Yoshida T., Nishida M., Nishida Y., Nasu K., and Narahara H. (2012) Bufalin, a Traditional Oriental Medicine, Induces Apoptosis in Human Cancer Cells. Asian Pacific Journal of Cancer Prevention, 13: 399-402.

7. Shao Jin-hua. (2010) Effects of Lobelia chinensis Lour Decoction on Expression of P53, C-erbB-2 and PCNA in H22 Tumor-bearing Mice. Dalian Medical University.

8. Shen Hong-xun, Lu Fei and Qian Jing-jing. (2003) Experimental study on the effects of compound Shenqitang on hepatic metastasis, nm23-H and blood CCK20mRNA expression in human colon cancer cell-inoculated nude mice. Chinese Association of Integrative Medicine: 5-9.

9. Su Jun, Tan Xing, Li Jin-tao and Yang Jun. (2007) Extraction of Alkaloids from Lobelia chinensis Lour and Their Inhibitory Effects on Growth of Stomach Cancer Cells. Journal of China West Normal University (Natural Science), 04: 311-313.

10. Winnie Lai Ting Kan, Chi Hin Cho, John A. and Rudd Lin G. (2008) Study of the anti-proliferative effects and synergy of phthalides from Angelica sinensis on colon cancer cells. Journal of Ethnopharmacology, 120(1): 36-43.

11. Zhao Li. (2010) Analysis of Difference between Colon Cancer and Rectal Cancer in Clinical Features, Molecular Mechanism of Oncogenesis. Peking Union Medical College.

12. Zhou Xin, Dong Li, Wu Da-zheng and Huang Bo. (2010) Effects of Coptis chinensis, Evodia rutaecarpa and their drugs on proliferation and apoptosis of colonic crypt cells in DMH-induced rats. Pharmacology and Clinics of Chinese Materia Medica, 03: 35-39. 